\title{
Application of a New Bicyclic Triaminophosphine Ligand in Pd-Catalyzed Buchwald-Hartwig Amination Reactions of Aryl Chlorides, Bromides and Iodides
}

Sameer Urgaonkar, Ju-Hua Xu, and John G. Verkade* Department of Chemistry, Gilman Hall, Iowa State University, Ames, Iowa, 50011-3111 jverkade@iastate.edu

\author{
SUPPORTING INFORMATION
}

TABLE OF CONTENTS

\author{
Contents \\ Page numbers \\ General Considerations \\ S2 \\ References for known compounds \\ S2-S6
}


General Considerations: All reactions were performed under an atmosphere of argon in oven-dried glassware. Toluene was collected from a Solvent Purification System and stored over $4 \AA$ A molecular sieves. ${ }^{1} \mathrm{H}$ and ${ }^{13} \mathrm{C}$ NMR spectra were recorded at 300 and 75.5 MHz, respectively. Thin-layer chromatography (TLC) was performed using commercially prepared 60 mesh silica gel plates and visualized with short wavelength UV light $(254 \mathrm{~nm})$. Silica gel 60 (9385, 230-400 mesh) was used for column chromatography. The yields reported are isolated yields and are the average of at least two runs. All commercially available reagents were used as received. For convenience, a stock solution of $\mathbf{3}$ in toluene $(2 \mathrm{mM})$ was prepared and stored under argon. All compounds described in Tables 1-4 are known in the literature and were characterized by comparing their ${ }^{1} \mathrm{H}$ and ${ }^{13} \mathrm{C}$ NMR or mass spectra to the previously reported data. In all cases, the comparisons were very favorable.

\section{References for known compounds:}

$N$-Methyl- $N$-(4-cyanophenyl)aniline (Table 1, entry 1). Stauffer, S. R.; Lee, S.; Stambuli, J. P.; Hauck, S. I.; Hartwig, J. F. Org. Lett. 2000, 2, 1423.

$N$-(4-tert-Butylphenyl)morpholine (Table 1, entry 2). Kataoka, N.; Quinetta, S.; Stambuli, J. P.; Hartwig, J. F. J. Org. Chem. 2002, 67, 5553.

$N, N$-Dibutyl-4-tert-butylaniline (Table 1, entry 3). Wolfe, J. P.; Tomori, J.; Sadighi, J. P.; Yin, J.; Buchwald, S. L. J. Org. Chem. 2000, 65, 1158.

$N$-(4-tert-Butylphenyl)diphenylamine (Table 1, entry 4). Creason, S. C.; Wheeler, J.; Nelson, R. F. J. Org. Chem. 1972, 37, 4440.

$N$-(4-tert-Butylphenyl)-p-anisidine (Table 1, entry 5). Wolfe, J. P.; Tomori, J.; Sadighi, J. P.; Yin, J.; Buchwald, S. L. J. Org. Chem. 2000, 65, 1158. 
$N$-(4-Methoxyphenyl)morpholine (Table 1, entry 6). Wolfe, J. P.; Buchwald, S. L. J. Org. Chem. 1996, 61, 1133.

$N$-(4-Methoxyphenyl)piperidine (Table 1, entry 7). Brenner, E.; Schneider, R.; Fort, Y. Tetrahedron 1999, 55, 12829.

N,N-Dibutyl-p-anisidine (Table 1, entry 8). Kataoka, N.; Quinetta, S.; Stambuli, J. P.; Hartwig, J. F. J. Org. Chem. 2002, 67, 5553.

N-(4-Methoxyphenyl)diphenylamine (Table 1, entry 9). Kataoka, N.; Quinetta, S.;

Stambuli, J. P.; Hartwig, J. F. J. Org. Chem. 2002, 67, 5553.

$N$-Methyl- $N$-(4-methoxyphenyl)aniline (Table 1, entry 10). Kataoka, N.; Quinetta, S.;

Stambuli, J. P.; Hartwig, J. F. J. Org. Chem. 2002, 67, 5553.

N-(4-Methylphenyl)-p-anisidine (Table 1, entry 11). Antilla, J. C.; Buchwald, S. L. Org. Lett. 2001, 3, 2077.

$N$-(2,6-Dimethylphenyl)-p-anisidine (Table 1, entry 12). Desmarets, C.; Schneider, R.; Fort, Y. J. Org. Chem. 2002, 67, 3029.

$N$-(2',4',6'-Trimethylphenyl)-2,6-dimethylaniline (Table 1, entry 13). Urgaonkar, S.;

Nagarajan, M.; Verkade, J. G. Org. Lett. 2003, 5, 815.

N-(2-Pyridyl)morpholine (Table 1, entry 14). Wolfe, J. P.; Tomori, J.; Sadighi, J. P.;

Yin, J.; Buchwald, S. L. J. Org. Chem. 2000, 65, 1158.

N,N-Dibutyl-2-aminopyridine (Table 1, entry 15). Wolfe, J. P.; Tomori, J.; Sadighi, J.

P.; Yin, J.; Buchwald, S. L. J. Org. Chem. 2000, 65, 1158.

N-(2-Pyridyl)diphenylamine (Table 1, entry 16). Kanemaru, T.; Nagahara, S.; Senoo, A. Eur. Pat. Appl. 449742, 1991; Chem. Abstr. 1991, 116, 204473. 
$N$-Methyl- $N$-(3-pyridyl)aniline (Table 1, entry 17). Urgaonkar, S.; Nagarajan, M.; Verkade, J. G. Org. Lett. 2003, 5, 815.

$\boldsymbol{N}$-(4-Nitrophenyl)morpholine (Table 2, entry 1). Kataoka, N.; Quinetta, S.; Stambuli, J. P.; Hartwig, J. F. J. Org. Chem. 2002, 67, 5553.

$N$-Methyl- $N$-(4-nitrophenyl)aniline (Table 2, entry 2). Kuwano, R.; Utsunomiya, M.; Hartwig, J. F. J. Org. Chem. 2002, 67, 6479.

$N$-( 3-Carbomethoxy)morpholine (Table 2, entry 3). Wolfe, J. P.; Tomori, J.; Sadighi, J. P.; Yin, J.; Buchwald, S. L. J. Org. Chem. 2000, 65, 1158.

$\boldsymbol{N}$-(3-Carbomethoxy)pyrrolidine (Table 2, entry 4). Semple, G.; Ryder, H.; Rooker, D. P.; Batt, A. R.; Kendrick, D. A.; Szelke, M.; Ohta, M.; Satoh, M.; Nishida, A.; Akuzawa, S.; Miyata, K. J. Med. Chem. 1997, 40, 331.

$N$-(4-Carbomethoxy)morpholine (Table 2, entry 5). Wolfe, J. P.; Tomori, J.; Sadighi, J. P.; Yin, J.; Buchwald, S. L. J. Org. Chem. 2000, 65, 1158.

$N$-(4-tert-Butylphenyl)-m-nitroaniline (Table 2, entry 6). Cundy, D. J.; Forsyth, S. A. Tetrahedron Lett. 1998, 39, 7979.

$N$-(4-Cyanophenyl)morpholine (Table 3, entry 1). Wolfe, J. P.; Buchwald, S. L. J. Am. Chem. Soc. 1997, 119, 6054.

$N$-(4-Cyanophenyl)-p-toluidine (Table 3, entry 3). Wolfe, J. P.; Tomori, J.; Sadighi, J. P.; Yin, J.; Buchwald, S. L. J. Org. Chem. 2000, 65, 1158.

$N$-(4-Nitrophenyl)-p-anisidine (Table 3, entry 4). Wolfe, J. P.; Tomori, J.; Sadighi, J. P.; Yin, J.; Buchwald, S. L. J. Org. Chem. 2000, 65, 1158.

$N$-(4-Nitrophenyl)diphenylamine (Table 3, entry 5). Gujadhur, R.; Venkataraman, D.; Kintigh, J. T. Tetrahedron Lett. 2001, 42, 4791. 
$N$-Methyl- $N$-(4-carbomethoxyphenyl)aniline (Table 3, entry 8). Kataoka, N.; Quinetta, S.; Stambuli, J. P.; Hartwig, J. F. J. Org. Chem. 2002, 67, 5553.

$N$-(3-Carbomethoxy)piperidine (Table 3, entry 10). Semple, G.; Ryder, H.; Rooker, D.

P.; Batt, A. R.; Kendrick, D. A.; Szelke, M.; Ohta, M.; Satoh, M.; Nishida, A.; Akuzawa, S.; Miyata, K. J. Med. Chem. 1997, 40, 331.

$N$-Methyl- $N$-(3-carbomethoxyphenyl)aniline (Table 3, entry 11). Wolfe, J. P.; Tomori, J.; Sadighi, J. P.; Yin, J.; Buchwald, S. L. J. Org. Chem. 2000, 65, 1158.

$N$-(4-Methylphenyl)morpholine (Table 3, entry 13). Wolfe, J. P.; Buchwald, S. L. $J$. Org. Chem. 1996, 61, 1133.

$N$-(3-Methoxyphenyl)morpholine (Table 3, entry 15). Kataoka, N.; Quinetta, S.; Stambuli, J. P.; Hartwig, J. F. J. Org. Chem. 2002, 67, 5553.

$N$-(3-Methoxyphenyl)piperidine (Table 3, entry 16). Desmarets, C.; Schneider, R.; Fort, Y. J. Org. Chem. 2002, 67, 3029.

$N$-(3-Methoxyphenyl)-p-toluidine (Table 3, entry 17). Beard, R. L.; Teng, M.; Colon, D. F.; Duong, T. T.; Chandraratna, R. A. U.S. Patent 5739338, 1998; Chem. Abstr. 1998, $128,282789$.

$N$-(4-Pyridyl)morpholine (Table 3, entry 19). Wolfe, J. P.; Tomori, J.; Sadighi, J. P.; Yin, J.; Buchwald, S. L. J. Org. Chem. 2000, 65, 1158.

$N$-Methyl- $N$-(4-pyridyl)aniline (Table 3, entry 20). Wolfe, J. P.; Tomori, J.; Sadighi, J. P.; Yin, J.; Buchwald, S. L. J. Org. Chem. 2000, 65, 1158.

$N$-Methyl- $N$-(4-chlorophenyl)aniline (Table 4, entry 1). Wolfe, J. P.; Buchwald, S. L. J. Org. Chem. 1996, 61, 1133. 
$N$-Methyl- $N$-(4-carboethoxyphenyl)aniline (Table 4, entry 2). Wolfe, J. P.; Buchwald, S. L. J. Org. Chem. 2000, 65, 1144.

N-(4-Carboethoxy)morpholine (Table 4, entry 3). Belfield, A. J.; Brown, G. R.; Foubister, A. J.; Ratcliffe, P. D. Tetrahedron 1999, 55, 13285.

$N, N$-Dibutyl-4-carboethoxyaniline (Table 4, entry 4). Wolfe, J. P.; Buchwald, S. L. Tetrahedron Lett. 1997, 38, 6359.

$N$-(4-Methylphenyl)-1,4-dioxa-8-azaspiro[4.5]decane (Table 4, entry 6). Wolfe, J. P.; Buchwald, S. L. J. Org. Chem. 1996, 61, 1133.

N-(4-Methylphenyl)-2',6'-Dimethylaniline (Table 4, entry 7). Urgaonkar, S.; Nagarajan, M.; Verkade, J. G. Org. Lett. 2003, 5, 815.

N,N-Dibutyl-p-toluidine (Table 4, entry 8). Wolfe, J. P.; Buchwald, S. L. J. Org. Chem. 1996, 61, 1133. 